\title{
Afirmando su justicia. El sistema vindicatorio shuar y el desarrollo de la justicia indígena*
}

\author{
Asserting their Justice. The Shuar Vindicatory System and \\ the Development of Indigenous Justice
}

\author{
Raúl Márquez Porras ${ }^{1}$ \\ Universidad de Barcelona \\ Enrique D. Luzuriaga Muñoz ${ }^{2}$ \\ Observatorio de Conflictos Socioambientales/ \\ Universidad Técnica Particular de Loja (Ecuador) \\ Carmen G. Puchaicela Huaca ${ }^{3}$ \\ Observatorio de Conflictos Socioambientales/ \\ Universidad Técnica Particular de Loja (Ecuador)
}

\section{RESUMEN}

En Ecuador, el reconocimiento de las justicias indígenas por el Estado se da en un escenario donde el marco legal ha sido solo parcialmente concretado, los distintos pueblos ancestrales discuten su propio modelo de justicia y mantienen una relación ambigua con la justicia estatal. Este artículo observa la realidad jurídica de uno de estos pueblos, los shuar, a partir de un estudio de caso en el cantón Nangaritza y de trabajo bibliográfico. En primer lugar, se caracteriza la justicia ancestral shuar, eminentemente vindicatoria. A continuación se describen algunas dinámicas de cambio y la relación, en general, de los shuar con los representantes de la justicia estatal. Y finalmente, se señalan algunas hipótesis sobre la adaptación de la justicia shuar al nuevo marco constitucional y su proceso de oficialización.

Palabras clave: Justicias indígenas; Shuar; Ecuador; Nuevo constitucionalismo; Pluralismo legal.

\section{SUMMARY}

In Ecuador, the State's recognition of indigenous justice systems takes place in a context where the legal framework is only partially made explicit, native communities debate their own legal

* La investigación en que se basa este artículo ha contado con el apoyo y financiación de la Secretaría Nacional de Educación Superior, Ciencia, Tecnología e Innovación de Ecuador, en la forma de una beca Prometeo (2016-009) disfrutada por el Dr. Márquez entre julio-octubre de 2016. Agradecemos, asimismo, la ayuda brindada por el Observatorio de Conflictos Socioambientales y el Departamento de Ciencias Jurídicas de la Universidad Técnica Particular de Loja, con mención especial para María Beatriz Eguiguren y Maritza Ochoa, quienes accedieron a compartir parte de su trabajo en Nangaritza con nosotros.

${ }^{1}$ Correo electrónico: raulmarquez@ub.edu. ORCID iD: <https://orcid.org/0000-0002-2765-1845>.

${ }^{2}$ Correo electrónico: edluzuriaga@utpl.edu.ec. ORCID iD: <https://orcid.org/0000-0002-0152-096X>.

${ }^{3}$ Correo electrónico: cgpuchaicela@utpl.edu.ec. ORCID iD: <https://orcid.org/0000-0001-9947-9303>. 
model and their relationship with the State legal system remains ambiguous. This paper addresses the legal realities of one of these groups, the Shuar, drawing on a case study carried out in Nangaritza and on bibliographic work. First, a characterisation of the Shuar traditional justice is attempted. Then, certain dynamics of change, as well as the relationship between the Shuar and the State's representatives, is described. Finally, hypotheses are proposed concerning the adaptation of Shuar justice to the new constitutional framework and its formalisation process.

Keywords: Indigenous Legal Systems; Shuar; Ecuador; New Constitutionalism; Legal pluralism.

\section{INTRODUCCIÓN}

En un proyecto estatal como el ecuatoriano, que busca construir un Estado plurinacional que respete e integre las distintas culturas y nacionalidades de su territorio, la institución de la(s) justicia(s) indígena(s) resulta un pilar fundamental. No en vano, señalan numerosos autores, preservar una cultura pasa por mantener su justicia, su manera de prevenir y resolver los conflictos y de organizar la vida social, en general; el derecho es, sin duda, "un elemento básico de la identidad étnica de un pueblo, nación o comunidad" (Stavenhagen 2006: 15).

Ecuador ha avanzado mucho en el establecimiento formal del pluralismo jurídico - como reconocimiento de otros sistemas de justicia operando dentro del espacio estatal (Grijalva 2012a; Santos 2012; Wolkmer 1994)_, de la mano de un constitucionalismo muy innovador. La Constitución de la República del Ecuador de 2008 reconoce a los pueblos y nacionalidades indígenas derechos específicos, colectivos, por el hecho de mantener rasgos culturales y formas de organización social particulares. Concretamente, el art. 171 faculta a las comunidades indígenas para administrar justicia aplicando sus normas y procedimientos propios — con la salvedad de que no atenten contra los derechos humanos y otros convenios internacionales ${ }^{4}$ :

Las autoridades de las comunidades, pueblos y nacionalidades indígenas ejercerán funciones jurisdiccionales, con base en sus tradiciones ancestrales y su derecho propio, dentro de su ámbito territorial, con garantía de participación y decisión de las mujeres. Las autoridades aplicarán normas y procedimientos propios para la solución de sus conflictos internos, y que no sean contrarios a la Constitución y a los derechos humanos reconocidos en instrumentos internacionales. El Estado garantizará que las decisiones de la jurisdicción indígena sean respetadas por las instituciones y autoridades públicas. Dichas decisiones estarán sujetas al control de constitucionalidad. La ley establecerá los mecanismos de coordinación y cooperación entre la jurisdicción indígena y la jurisdicción ordinaria.

No obstante, esa coordinación con la justicia estatal que señala la Constitución (y el Convenio 169 de la Organización Internacional del Trabajo, suscrito por Ecuador

\footnotetext{
${ }^{4}$ Asimismo, el art. 343 del Código Orgánico de la Función Judicial dispone: "Las autoridades de las comunidades, pueblos y nacionalidades indígenas ejercerán funciones jurisdiccionales, con base en sus tradiciones ancestrales y su derecho propio o consuetudinario, dentro de su ámbito territorial, con garantía de participación y decisión de las mujeres. Las autoridades aplicarán normas y procedimientos propios para la solución de sus conflictos internos, y que no sean contrarios a la Constitución y a los derechos humanos reconocidos en instrumentos internacionales. No se podrá alegar derecho propio o consuetudinario para justificar o dejar de sancionar la violación de derechos de las mujeres".
} 
en 1998), aún no ha sido establecida ${ }^{5}$. Y su ausencia complica el ejercicio efectivo de las justicias indígenas. Por el momento, la Corte Constitucional ha actuado como coordinador de facto al presentarse algunos conflictos, con fallos que han sido muy controvertidos y han representado un cuestionamiento de la autonomía de la justicia indígena, de su alcance y procedimientos — véase el conocido caso de La Cocha, entre 2010 y 2014, en el que la Corte revisaba la resolución de un caso de homicidio en una comunidad kichwa panzaleo (cf. Aparicio 2015; Grijalva y Exeni 2012; Stavenhagen 2006) - Comentan algunos autores que esta situación se debe, en realidad, a ciertos prejuicios etnocéntricos que aún tienen los legisladores, que les llevan a considerar las justicias indígenas como inferiores, poco evolucionadas e irracionales (Chávez y García 2004: 200; Grijalva 2012b: 555-575; Santos 2012: 18-31).

De hecho, se constata una comprensión bastante deficitaria de las culturas jurídicas indígenas — de algunas más que de otras-, lo que explica las dudas en cuanto a su efectivización. Para empezar, resulta una simplificación obvia hablar de justicia indígena en singular, como si solo existiera un tipo o fuese igual en todos los pueblos y nacionalidades. Las justicias indígenas son diversas entre ellas en cuanto a instituciones, procedimientos, sanciones, etc. Quizás lo único que tienen en común es "el hecho de ser ejercidas en las comunidades por autoridades propias y reconocidas" (Santos 2012: 21). Además, como pasa con cualquier otro ámbito de una cultura, se transforman y modifican constantemente; por lo que deberían ser estudiadas etnográficamente, para captar el significado actual de sus instituciones y su estado de funcionamiento (Grijalva 2012a: 67). El hecho es que los antropólogos/as -a quienes correspondería dicha tarea- no se han detenido demasiado en el campo de lo jurídico. Mientras que los juristas, por su parte, se han dedicado más al estudio de los sistemas estatales (Stavenhagen 2006: 15).

En el caso de Ecuador, tampoco abundan los estudios sobre los sistemas de justicia de sus pueblos y nacionalidades. Y los existentes versan sobre algunas nacionalidades concretas, preferentemente la kichwa (Ávila 2012; Brandt y Franco 2007; Vintimilla, Almeida y Saldaña 2007). Sobre los pueblos de las tierras bajas amazónicas, como los shuar, prácticamente no hay nada. Y las pocas investigaciones sobre ellos dan, además, interpretaciones bastante inadecuadas, como veremos. El establecimiento de un verdadero pluralismo jurídico, del pleno reconocimiento estatal de los sistemas de justicia indígenas, requeriría, en cambio, de un estudio pormenorizado de las diversas culturas jurídicas (Chávez y García 2004: 211; Fiallo 2014: 14-16; Santos 2012).

Cabe decir, asimismo, que es una demanda de los propios pueblos indígenas el que se investigue su cultura jurídica ancestral, con el objetivo de sistematizar y fortalecer sus sistemas de justicia (Llasag 2012: 366). Justamente los shuar de Nangaritza, sus organizaciones representativas, están iniciando un proceso de sistematización y

\footnotetext{
${ }^{5}$ Ha habido proyectos de esta ley de coordinación entre sistemas de justicia, como el presentado por la congresista Lourdes Tibán en 2010. E informes de la Comisión de Justicia y Estructura del Estado sobre dicha Ley de Coordinación y Cooperación entre los Sistemas de Justicia Indígena y la Jurisdicción Ordinaria (Grijalva y Exeni 2012: 595-596). Sin que se haya avanzado en el asunto a fecha de hoy, topando proyectos como el de Tibán con el veto del Congreso. Aprovechamos para comentar nuestra preferencia por el término justicia estatal antes que ordinaria, ya que este último connotaría que las otras justicias son extraordinarias: o sea, excepcionales, extrañas, absolutamente distintas.
} 
búsqueda de reconocimiento de su justicia. En dicho proceso se inserta este texto, que es, básicamente, una exploración general de la justicia ancestral shuar y de algunas de sus dinámicas actuales. Su interés estriba, creemos, en el propio hecho de ser un estudio empírico e interdisciplinar — desde el derecho y la antropología - sobre una cultura jurídica indígena específica, hasta el momento mal comprendida.

Los shuar (llamados jíbaros por los conquistadores españoles, término que aludía a su presunto salvajismo) son caracterizados en la literatura como un pueblo sin derecho $^{6}$. Supuestamente propensos a la violencia, las percepciones en torno a su agresividad y falta de control abundan en la literatura: "Los Jívaros son por naturaleza impulsivos y coléricos", decía en 1935 uno de sus primeros etnógrafos (Karsten 1988 [1935]: 307). Un saraguro, informante de otro antropólogo, contaba de un amigo shuar: "Aunque Mariano había vivido afuera por un tiempo, era un verdadero jíbaro. En verdad le gustaba matar a la gente" (Karsten 1988 [1935]: 288). Más importante aún, se representa a los shuar como carentes de un sistema de justicia estructurado (si entendemos por esto el que cuenta con normas colectivas y consensuadas, cierta regularidad y procedimientos sancionadores claros). Comentaba Michael Harner:

the Jivaro apply sanctions against alleged violators of the norms purely by means of informal partisan action (...). The determination of guilt in a specific case and of the proper sanction for a specific offense is a rather individualistic matter, with a significant degree of variability (1972: 171).

En realidad, autores como Harner son bastante ambivalentes: sí parecen reconocer una cierta lógica y sistematicidad en la forma de resolver los conflictos -y de ordenar la vida social, en general- por parte de los shuar. La describen en detalle, de hecho. Pero entienden erróneamente algunos de sus elementos, y al final parecen negar aquello que describen, como veremos en el siguiente apartado.

Por otro lado, y aunque nos dediquemos en parte a analizar su forma ancestral, la justicia shuar no se ha mantenido inalterada a lo largo del tiempo. Ha ido adaptándose a circunstancias históricas concretas (Chenaut 2006: 51; Grijalva 2012b: 575576; Santos 2012: 30; Sierra y Stavenhagen 2006: 19). Y, como pasa con el resto de instituciones y en todos los pueblos, no todos los shuar la entienden de la misma manera, ni tienen la misma capacidad para interpretar y utilizar sus componentes (Chávez y García 2004: 211; Roseberry 2014; Rubenstein 2005: 30).

Uno de los factores de cambio y desigualdad más importantes es la interacción con el sistema de justicia estatal. A lo cual concede mucha importancia la teoría de plura-

\footnotetext{
${ }^{6}$ Esta minusvaloración de los sistemas de justicia indígenas es algo general, como decíamos, que algunos autores atribuyen a perspectivas etnocéntricas o directamente racistas: "según esta visión racista el Derecho Indígena es un no-Derecho, un cúmulo de prácticas arcaicas violatorias de los más básicos derechos humanos y derechos constitucionales. Desde esta perspectiva, la justicia indígena es una suerte de legado ancestral y bárbaro que ha permanecido estático por siglos sin recibir la influencia civilizadora del verdadero Derecho, el Derecho occidental" (Grijalva 2012b: 575). Los pueblos kichwa de Zamora Chinchipe lo denuncian así: "el 99\% de las ecuatorianas y ecuatorianos siguen pensando que justicia indígena es ortigar, bañar con agua fría y dar unos cuantos azotazos"; y atribuyéndose, al parecer, cierta responsabilidad, comentan: "Esto sucede porque no hemos sido capaces de institucionalizar la Justicia Indígena; por lo menos de la Nacionalidad Kichwa; porque de los sistemas de justicia de las otras nacionalidades casi nada o muy poco sabemos" (GAD Provincial Zamora Chinchipe 2016: 13-14).
} 
lismo jurídico que aquí seguimos. Es evidente que en la actualidad no se puede estudiar un sistema de justicia indígena como opuesto a y radicalmente separado del estatal; ambos conviven en espacios configurados, principalmente, por los Estados. Lo que abundan son las situaciones de pluralismo, los intercambios, préstamos e hibridaciones (Chávez y García 2004; Fiallo 2014; Santos 2012). Interlegalidad es un concepto acuñado para referirse a esa constitución mutua entre sistemas (Santos 2012; Sierra y Chenaut 2006). Y en antropología resulta clásica la concepción de los sistemas jurídicos como campos semiautónomos: instituciones, prácticas sociales que son solo parcialmente autónomas y autorreguladas, que en la práctica se superponen y entremezclan con otras (lo informal con lo más o menos formal, las regulaciones locales con las estatales, etc.) (Moore 1972; Fiallo 2014). Justamente, lo interesante es estudiar esas superposiciones, los híbridos resultantes de la relación (en nuestro caso) entre el sistema jurídico shuar y los otros sistemas y culturas presentes en el área donde actúa.

Así, esta investigación se interroga, entre otras cosas, sobre la cultura jurídica que tenían y tienen los shuar de Nangaritza; sobre las hibridaciones y reactualizaciones de dicha justicia en el marco del nuevo constitucionalismo y la relación contemporánea con el Estado ecuatoriano; y sobre las expectativas y percepciones que los shuar tienen respecto a esa relación y al ejercicio de su propia justicia.

A nivel metodológico, este texto se basa en un estudio de caso realizado en algunas comunidades del cantón Nangaritza (provincia de Zamora Chinchipe, suroriente ecuatoriano). Principalmente en Yayu (parroquia de Nuevo Paraíso), pero también en otras comunidades shuar de la zona y en la comunidad pluriétnica de Nuevo Paraíso, que justamente por su composición multiétnica servía de contrapunto a Yayu. Parte del equipo que ha elaborado este texto lleva desde 2013 trabajando en Nangaritza, analizando conflictos socioambientales. Entre julio y octubre de 2016 realizamos una investigación intensiva sobre culturas jurídicas, la cual incluyó visitas semanales a las comunidades, observación in situ y dos talleres sobre justicia indígena y manejo de los conflictos en Yayu y Nuevo Paraíso, respectivamente. Este trabajo sobre el terreno se complementó con entrevistas semiestructuradas a informantes clave (de la Federación shuar de la provincia de Zamora Chinchipe -FEPNASH—, de la Federación Provincial de la Nacionalidad Kichwa Saraguro -ZAMASKIJAT - y de las propias comunidades), con exploración en los archivos de la Fiscalía de Asuntos Indígenas de Zumbi, y con análisis de otra documentación y bibliografía.

Caracterizando mínimamente las comunidades foco del estudio, tanto Yayu como Nuevo Paraíso se sitúan en la margen del río Numpatakaime (un afluente del Nangaritza, y a su vez del Zamora). El río es la principal vía de comunicación - la carretera sin asfaltar llega solo hasta Nuevo Paraíso, sede del gobierno parroquialy el paisaje se encuentra dominado por los bosques siempreverdes propios de esta región amazónica. Nuevo Paraíso fue fundada en 1979 por emigrantes provenientes de la provincia de Loja. Actualmente viven en ella algo más de 200 personas, a partes iguales mestizos y shuar, pero también una minoría de origen kichwa saraguro (GAD Parroquial Nuevo Paraíso 2015). En Yayu se cuentan oficialmente unos 80 habitantes, la totalidad identificados como shuar. Yayu surgió a principios de los años 70 (s. XX), tras la llegada desde el norte de algunas familias desplazadas por la colonización mestiza (GAD Parroquial Nuevo Paraíso 2015; Melanya Anguash, entrevista personal, 21 sep. 2016). Estas familias, emparentadas entre sí, construirán sus casas 
de manera agrupada alrededor de una explanada rectangular (patrón de asentamiento que no corresponde al tradicional, como se comenta en el apartado sobre transformaciones contemporáneas).

Gran parte del territorio de ambas comunidades es propiedad colectiva e indivisa, bajo un título global, y cuenta con algún grado de protección ambiental. Las familias viven de la ganadería, la caza y la pesca, y del cultivo de productos para el consumo propio (yuca, plátano, maíz, etc.) y la venta (principalmente naranjilla), en pequeños huertos (fincas) que cada una tiene fuera del núcleo habitado. Algunas también se dedican a la extracción de madera y/o a la pequeña minería artesanal, de manera no siempre legal (Observatorio de Conflictos Socioambientales 2013). El contacto con el pueblo de Guayzimi, cabecera cantonal, y con las ciudades de Zamora e incluso Loja es regular, yendo los vecinos a realizar trámites, hacer uso de los servicios públicos y algunos, sobre todo jóvenes, a trabajar o estudiar ${ }^{7}$.

Realizada esta pequeña contextualización, la estructura del texto que sigue es la siguiente: en primer lugar, se describe la justicia ancestral shuar, de tipo vindicatorio, ahondando en su lógica y señalando algunas confusiones y malentendidos. A continuación, se presentan algunos cambios históricos que han afectado de manera fundamental la organización social shuar. En el siguiente apartado se abordan algunas dinámicas contemporáneas referidas, concretamente, a la práctica de la justicia en las comunidades estudiadas. Se tratan las expectativas y proyectos de los shuar en torno al desempeño de su justicia, incluyendo la comparación e interacción con otros sistemas jurídicos. Y, por último, se formulan algunas hipótesis sobre el futuro de dicho proyecto de justicia propia.

\section{LA JUSTICIA SHUAR: UNA JUSTICIA DE TIPO VINDICATORIO}

La cultura jurídica ancestral shuar se ajusta al modelo vindicatorio, tal y como ha sido sistematizado por algunos analistas y observado en múltiples sociedades aestatales (Terradas 2008 y 2012). Estos eran tradicionalmente sus componentes principales en cuanto a actores, normas, procedimientos y sanciones ${ }^{8}$ :

\footnotetext{
${ }^{7}$ Cabe señalar que la mayor parte de los shuar del Ecuador - también habitan territorio oficialmente del Perú-, unos 80.000 según el Censo del 2010, reside en la provincia norteña de Morona Santiago. No obstante, en Zamora Chinchipe constituyen la principal nacionalidad indígena, siendo en el cantón Nangaritza un 26\% del total de población (algo más de 1.370 personas) y en la parroquia de Nuevo Paraíso más del 54\%, unos 570 vecinos (GAD Provincial Zamora Chinchipe 2015; GAD Cantonal Nangaritza 2015; GAD Parroquial Nuevo Paraíso 2015). Hablando de Morona Santiago resulta obligado mencionar los graves conflictos que se han dado allí en torno a las grandes explotaciones mineras impulsadas por el Estado, explotaciones que han topado con la oposición de organizaciones shuar. Sin que podamos dedicarle aquí el espacio necesario, cabe apuntar que estos conflictos parecen indicar una contradicción de base entre el proyecto económico del Estado, basado en gran medida en el extractivismo, y los preceptos constitucionales que prevén la concesión de una gran autonomía a las comunidades indígenas (cf. Aparicio 2015; Martínez Novo 2014).

${ }^{8}$ Para esta reconstrucción nos basamos en el taller realizado en la comunidad de Yayu, el 17 de septiembre de 2016, en las entrevistas a Gabriel Awari (actual presidente de la FEPNASH) y a otros informantes clave, y en la bibliografía publicada sobre el tema, sobre todo en Harner (1972) y Chávez y García (2004).
} 
Cometida una ofensa por alguien, era culpabilizada la persona y su grupo familiar, así como agraviados la víctima directa y sus parientes. De este modo, los actores en la justicia shuar no eran individuos sino colectivos, y las responsabilidades eran grupales. A la hora de resarcir una ofensa:

The aggrieved party, together with those relatives willing to support him, takes upon himself the responsibility for punishing the person or persons he believes to be guilty of an offense against him or a close member of his family (Harner 1972: 171)9.

En caso de envenenamiento causado por una mujer, por ejemplo, se intentaba envenenar al hermano de esta. O cuando un adúltero se fugaba, se castigaba al hermano o pariente masculino más cercano (entrevista grupal, 17 sep. 2016).

Los hijos podían ofender a sus padres: quejándose de lo que comían, desobedeciéndoles o avergonzándoles de alguna manera (manteniendo relaciones con otra mujer cuando ya estaban comprometidos, por ejemplo). En tales casos se les administraban ciertas plantas que "purifican y dan energía", que "dan otra visión y ayudan a tener otra actitud": el guanto, el natem, la guayusa, etc. Se les hacía ayunar, y se les llevaba a la cascada, donde permanecían durante una semana. A la vuelta llevaban una dieta especial y se les seguían administrando las plantas purificadoras. En cierto momento, además, debían "comprometerse" ante sus padres a no volver a cometer la falta en cuestión. El objetivo de todo el proceso era "componerlos", "sacarles lo que tuvieran en la cabeza" (Yayu, entrevista grupal, 17 sep. 2016).

Toda una serie de normas regulaban las uniones matrimoniales y los contactos sexuales. En caso de saltarse la prohibición de mantener relaciones antes del matrimonio, los prometidos eran castigados por el padre de la chica, que los hería con el machete (Harner 1972: 175). Los padres del chico, además, le hacían ayunar "para que cogiera buena energía" y que cuando se casara "fuera alguien: capaz de contruir su casa, su canoa, ser buen cazador y pescador, y que no deje que ninguno de sus hijos se vea mal" (Yayu, entrevista grupal, 17 sep. 2016). El robo de una esposa, o el adulterio, eran ofensas más graves. Implicaban que el esposo agraviado podía machetear al amante de su esposa — sin matarlo-para "desquitarse" y "calmarse las iras"; con posterioridad los "mayores" de las dos familias negociaban la "paz" (Yayu, entrevista grupal, 17 sep. 2006). Si el culpable huía, el castigo recaía en un pariente masculino (Chávez y García 2004; Harner 1972: 175). Pero, importante, las macheteadas debían realizarse siempre delante de un tercero que pudiera defender al castigado en caso de exceso (Yayu, entrevista grupal, 17 sep. 2006).

Incesto se consideraba la relación con un pariente no clasificado como wahe (primos cruzados y parientes distantes); siendo el más serio el cometido entre miembros de la familia conyugal. En caso de incesto del padre con la hija, un wahe de esta (primo cruzado u otro potencial marido) tenía derecho a pegar al padre, que además debía dejar la casa familiar y era abandonado por la esposa. En caso de incesto entre hermanos, otro hermano les cortaba la cabellera y podía expulsarlos del vecinda-

\footnotetext{
${ }^{9}$ Sobre esta vinculación grupal, y hablando en general de ontología shuar, Karsten (1988 [1935]: 311) afirma que entre ellos "el individuo forma una parte inseparable del todo, a saber de la familia o tribu a la que pertenece", sin que se tenga "concepción de la personalidad individual".
} 
rio - en ausencia de un hermano lo hacía el padre o un primo cruzado- (Harner 1972: 177).

Los robos eran infrecuentes, tanto que su reparación no estaba sistematizada en la justicia ancestral shuar (Harner 1972: 178-179) — cabe relacionarlo con su modo de vida y la ausencia de una noción de propiedad privada ${ }^{10}$ - Pero sí existían ciertas reglas de herencia o transmisión de bienes. El hermano mayor de un hombre fallecido, por ejemplo, tenía derecho a heredar su esposa o esposas —entre los shuar se daba la poligamia-. Si no tenía hermanos lo hacía el primo paralelo de más edad (Harner 1972: 178). También se heredaban los bienes personales. Con la costumbre de repartir algo entre los hermanos a medida que llegaran a la edad adulta — sin que esto fuera una obligación en sentido estricto- (Harner 1972: 178-179).

Respecto a los homicidios —raramente se atribuían las muertes a causas naturales-, estos podían darse mediante violencia física (incluido el envenenamiento), la acción de hechizos o de espíritus vengadores (muisak). Según Harner (1972: 172), solo en los dos primeros casos se identificaba a un responsable vivo y se consideraba la acción de venganza. Para quien había embrujado a un familiar, la muerte era a través de hechicería (de violencia física si era un reincidente) (Harner 1972: 173).

Precisamente el tema de las venganzas es complejo. En primer lugar, los shuar distinguían la venganza de la guerra; la guerra (que incluía las famosas expediciones de toma de cabezas) se daba solo contra otras tribus, enemigas: los que uhablaban diferente" y "no eran familia" (Harner 1972: 182-187; Yayu, entrevista grupal, 17 sep. 2016). La venganza, como retaliación ante alguna ofensa, sí se producía en el interior de las comunidades. Lo importante, además, es que las venganzas (o, mejor dicho, retaliaciones) tenían su procedimiento. Había ciertas formalidades para iniciarlas; el "envío de mensajes a través de amigos", por ejemplo (Karsten 1988 [1935]: 310; Yayu, entrevista grupal, 17 sep. 2016). Interesante la descripción de Harner (1972: 181) al respecto [énfasis nuestro]:

The initiation of a feud does not formally occur until one of the parties sends the message to the other, "Let us fight with guns..." (...). It is a public statement of the legal justification for an attack at some future time.

Las retaliaciones no eran un asunto arbitrario y de violencia sin control, por lo tanto. Eran un "desquite" ante una ofensa grave, generalmente un homicidio. Desquite que permitía después negociar una reconciliación entre las familias (Yayu, entrevista grupal, 17 sep. 2016). Normalmente acababan cuando se mataba a un hombre mayor (un untä) de alguno de los bandos, por lo general el padre o el suegro del ofensor (Harner 1972: 182). Las familias volvían a enviarse entonces un mensaje, se-

\footnotetext{
${ }^{10}$ Ausencia parcial, referida a la propiedad sobre el territorio, no sobre ciertos objetos personales, manufacturados. Comentaba Harner: "Land, or natural resources, is not owned individually or communally, except in the sense that a specific garden or part of a garden is owned by a woman as long as it is being cultivated, usually no more than about five years. Even in this case, it is the capital improvement of the land that is owned, in the form of the garden, not the land in its natural state. No territorial boundaries are recognized for hunting, fishing, or other purposes on a tribal, neighborhood, or household level" (Harner 1972: 179). Esto habría cambiado a partir de la actuación de los misioneros, del establecimiento de los centros comunitarios y del sistema de propiedad privada en el territorio, según detalla Rubenstein (2005).
} 
gún Harner (1972: 182) algo como: "Let us stop killing. Now all is paid because you have killed and we have killed. Terminando las hostilidades sin que hubiera ningún ritual o celebración especial, reconciliadas las familias pero sin tener "confianza" la una en la otra (Yayu, entrevista grupal, 17 sep. 2016).

Otra manera de concluir la retaliación era a través de una compensación, de algún pago a la parte ofendida (normalmente, un rifle o algún animal). Chávez y García (2004: 94) señalan lo importante en este instrumento: es un pago de valor básicamente moral, que se produce tras la negociación entre las partes y por el que los ofensores reconocen la infracción y el daño causado. Harner (1972: 182) añade: "Feuds can be formally ended by payment, provided that the last killing occurred sufficiently long ago for the deceased's relatives to be willing to settle for less than a retaliatory killing ". El pariente masculino más cercano a la víctima era quien recibía el pago, y correspondía a los dos hombres mayores de cada familia informar y convencer al resto de que el enfrentamiento había terminado (Harner 1972: 182). También podía producirse la compensación en caso de adulterio si había pasado el tiempo suficiente; entregando el ofensor al marido ofendido una cinta de plumas de tucán (Harner 1972: 175-176).

Como autoridades en la justicia shuar, los padres actuaban como jueces ante los hijos, los hombres mayores de cada familia como árbitros o mediadores en caso de conflicto interfamiliar, y amigos de prestigio, chamanes y guerreros reconocidos como intermediarios en algunos casos internos y entre comunidades (Presidente FEPNASH, entrevista personal, 6 sep. 2016).

Todos los elementos hasta aquí descritos son típicos de una justicia de tipo vindicatorio. Por lo demás, y como apuntábamos en la introducción, no siempre bien representados o comprendidos. En efecto, las descripciones del sistema de justicia ancestral shuar —escasas - se muestran ambivalentes respecto a su funcionamiento y al significado de sus componentes. El propio Harner (1972: 170)—pese a las descripciones apuntadas - afirma que los shuar carecen de cualquier autoridad: "a society where there is no state, no chiefdom, no corporate kin groups, and, really, no locus of authority which can impose judicial decisions and enforce them". E incluso de normas establecidas; los shuar solo tendrían "relatively clear-cut and consensually approved rules" (1972: 171) —aunque en otros pasajes hable de "Jívaro norms", "approved sanctions" y "equivalent retaliation" (1972: 172-173)—.

La venganza (retaliación) tampoco ha sido bien entendida. Aparece en las descripciones como la anti-justicia, el paradigma de la falta de proceso: es violencia descontrolada, castigo unilateral, etc. Autores contemporáneos como Chávez y García (2004: 86), por ejemplo, afirman: «En términos regulatorios, la venganza antes que un mecanismo de justicia intra o interfamiliar era, precisamente, una señal de carencia o de falla de otros mecanismos de resolución de conflictos". Y lo mismo ocurre con las compensaciones. Un etnógrafo pionero de los shuar, Karsten, las describía como una especie de compra del silencio y el olvido. Comentaba: "Los Jívaros no encuentran nada de repugnante en estimar de este modo la vida de un pariente con un equivalente material" (Karsten 1988: 310). Aunque añadía que no funcionaban para delitos de sangre recién cometidos, cuando la indignación de la familia ofendida no podía ser comprada (Karsten 1988 [1935]: 311).

Estudiosos de los sistemas vindicatorios como Ignasi Terradas, en cambio, sí han iluminado esta modalidad de justicia, ayudándonos a comprender su funcionamiento 
y sentido. En síntesis, las justicias vindicatorias se ocupan de ofensas y de la obligación de reparación que estas crean. La responsabilidad se atribuye a grupos familiares, es corporativa, como apuntábamos. Y los grupos deben, justamente, reparar, volver a recomponer sus relaciones, reconciliarse, dejándolo no a la actuación libre de las partes, sino incluyendo la intercesión de alguna autoridad, un tercero que más o menos media, arbitra o adjudica. Cuando se incluyen ofrecimientos materiales, compensaciones, su sentido no es comprar a la familia de la víctima sino reconocer que la deuda contraída es impagable; y no es posible hacerlo antes de que haya habido reconciliación. Es un tipo de justicia impregnada de consideraciones morales y religiosas, por lo demás (Terradas 2008 y 2012).

La cultura jurídica ancestral de los shuar contenía estos rasgos, como se ha visto: se trataba de una justicia incrustada en la vida social y familiar general, orientada a la superación del conflicto y, sobre todo, a la reconciliación entre las partes - grupos familiares-. Que se dedicaba a purificar al infractor (mediante el ayuno, el consumo de ciertas plantas...), y no solo a castigarlo. Que incluía como paso fundamental la reparación de la ofensa y el reconocimiento del daño causado, mediando la intervención de un tercero (un amigo, un hombre mayor de cada familia, el chamán o el guerrero reconocido), y en ocasiones el pago de una compensación para ratificar ese reconocimiento y el restablecimiento de las relaciones. Una justicia que establecía toda una serie de procesos, de actuaciones pautadas y regulares, en cada una de las fases de la resolución de los conflictos (enviar el mensaje antes de la retaliación, la presencia de un tercero durante la aplicación del castigo físico, etc. $)^{11}$.

\section{TRANSFORMACIÓN DE LOS SHUAR Y PRÁCTICAS JURÍDICAS CONTEMPORÁNEAS}

Los shuar no viven de la manera en que lo hacían y, por lo tanto, tampoco practican exactamente el mismo tipo de justicia hasta aquí descrito. El gran cambio contemporáneo ha sido, según numerosos autores, el establecimiento en centros (o comunidades) — hábitats agrupados y más o menos permanentes- y el surgimiento de nuevos liderazgos (Chávez y García 2004; Harner 1972; Rubenstein 2005) ${ }^{12}$.

Como es típico de los pueblos organizados en bandas (cazadores-recolectores en su mayoría), los shuar tenían líderes circunstanciales, ancianos sabios o guerreros que comandaban determinadas actividades (Clastres 1978). En cambio, desde mediados del s. XX — de la mano de la acción misionera y estatal—, surge la figura del síndico,

\footnotetext{
${ }^{11}$ Elementos que también están presentes en otras justicias indígenas del Ecuador, igualmente vindicatorias, como describen Ávila (2012), Trujillo (2012), Llasag (2012), Stavenhagen (2006), Grijalva (2012b), Grijalva y Exeni (2012), Chávez y García (2004), García (2002) o Fiallo (2014).

${ }^{12}$ A Harner le impresionaron estos cambios, al punto de afirmar que: "There are probably few cultures in the history of the world that have been so rapidly and significantly disintegrated by the simple introduction of centralized "law and order" (Harner, 1972: 210). Mientras que Rubenstein (2005: 30) afirmaba que se trataba de un cambio "de tal naturaleza que solo puede suceder una vez; el cambio que demarca el paso de un orden social a otro". No podemos detenernos a comentar otros cambios que van de la mano de los citados, como la creciente participación en la economía de mercado, que Rubenstein (2005: 44) ya observaba estaba "exacerbando e institucionalizando diferencias en riqueza material e influencia entre los shuar".
} 
que representa y lidera una comunidad, y de las directivas de asociaciones y federaciones, que ostentan un poder político considerable. A ellos se sumaría el presidente de la junta parroquial y otros cargos de la administración, o incluso los catequistas (Chávez y García 2004: 86; Rubenstein 2005). Los nuevos líderes son en su mayoría jóvenes - lo cual revierte la pauta tradicional-, formados en escuelas misioneras y que, entre otras cosas, hablan mejor el español que sus padres (Rubenstein 2005: 39). Organizados jerárquicamente en asociaciones y federaciones, los shuar participan de las instituciones estatales y conocen sus leyes (Rubenstein 2005: 41-42) ${ }^{13}$.

En las comunidades estudiadas se constata dicha estructura de autoridad: por ejemplo, en Yayu el síndico —un hombre joven- es la figura más relevante, junto con un hermano que preside una de las asociaciones shuar - escisión reciente de otray algunos hombres que han recibido instrucción en escuelas de colonos. La presidenta de la parroquia de Nuevo Paraíso, de la que forman parte, también ejerce cierta autoridad. No obstante, los mayores de la comunidad mantienen cierto peso y son escuchados en la decisión de los asuntos importantes. La asociación de la cual forma parte Yayu está integrada, a su vez, en la federación provincial shuar (FEPNASH), organización legalizada por el Estado y convertida en uno de los portavoces oficiales del pueblo shuar.

Todos estos cambios impactan, como decíamos, en el sistema de justicia shuar. En la mayor parte de comunidades los síndicos cumplen funciones de juez (árbitro o mediador), funciones que están estipuladas, incluso, en los estatutos de algunas comunidades y asociaciones (Chávez y García 2004). En la mayor parte de comunidades del cantón Nangaritza, en efecto, el síndico es una primera instancia en la resolución de los conflictos, con poder para hacerse escuchar por las partes y, dependiendo del caso, recomendar o imponer resoluciones y castigos. Las otras autoridades funcionan como distintas instancias de apelación. Así, si un problema no se consigue resolver a nivel de comunidad, pasa a la directiva de la asociación (o asociaciones) en que está inscrita la comunidad. Y si tampoco allí se consigue solucionar, se llama a los responsables de las federaciones de zona para que intervengan (Chávez y García 2004: 88-89; Presidente FEPNASH, entrevista personal, 6 sep. 2016).

En términos concretos, el sistema de justicia shuar ha perdido uno de los elementos centrales como eran las retaliaciones entre familias, al ser prohibidas estas por el Estado y los misioneros; según Harner, a finales de la década de 1960 el Estado llegó a encarcelar a quienes participaban en ellas (1972: 210). Un vicepresidente de la Federación Interprovincial de Centros shuar (FICSH), declaraba: "Los misioneros nos obligaron a que dejemos nuestras costumbres (...); ahora no podemos hacer eso [ven-

\footnotetext{
${ }^{13}$ Recordamos que antes eran autoridad, además de los mayores de las familias, el chamán o chamanes y los guerreros más reputados. De los primeros hoy se dice que abundan los "malos chamanes", que solo trabajan para "enemistar" y no siguen el procedimiento para formarse — consistente en ayunar y abstenerse de relaciones sexuales durante un año para recibir "fuerza", básicamente- (Presidente de la FEPNASH, entrevista, 6 sep. 2016). Respecto a los guerreros, el fin de las guerras entre tribus (tras la instauración del control estatal sobre el territorio) habría extinguido tal figura. En general, existe cierto malestar por no tener líderes más "fuertes", que además se preparen como era la costumbre (tomando regularmente natem, etc.), en lo que es expresión de cierto malestar (Chávez y García 2004: 97; Yayu, entrevista grupal, sep. 2016).
} 
ganzas] porque existen las leyes del Estado" (Chávez y García 2004: 86). Tanto es así que la XXX Asamblea de la FICSH, en 1996, decidió substituir en su reglamento interno la sanción de muerte por venganza, por la de expulsión de la comunidad y enajenación de los bienes ${ }^{14}$. En las comunidades de Nangaritza, por su parte, se asume que "la ley del Estado ya no permite aplicar la ley de matarse" (Yayu y Nuevo Paraíso, entrevistas grupales, sep. 2016). Otras prácticas puntuales también han sido perseguidas, como el consumo de substancias psicotrópicas (natem, guanto...). Según vecinos de Yayu, los misioneros les decían en la escuela que no las tomaran porque "Se volverían tontos" (entrevista grupal, 17 sep. 2016).

Según algunas etnografías recientes y lo observado en Nangaritza, hay ofensas contempladas tradicionalmente cuya frecuencia ha aumentado: por ejemplo, los casos de brujería. Esto se atribuye al número creciente de malos chamanes (Chávez y García 2004: 203; Harner 1972: 201; Presidente de la FEPNASH, entrevista personal, 6 sep. 2016). También habría cambiado el hecho que antes se hacía responsable a toda la familia del chamán, y hoy no (se toman represalías solo contra él) (Chávez y García 2004: 101).

También aumentan las ofensas personales, provocadas generalmente por la incontinencia de personas embriagadas - un problema extendido en las comunidades es la excesiva ingesta de alcohol- Estos casos son resueltos por los síndicos de las comunidades con cierta facilidad (Chávez y García 2004: 203). Y son también habituales los conflictos por chismes o habladurías, donde se obliga al ofensor a que pida disculpas públicamente y realice ciertos trabajos comunitarios (elementos típicos de una justicia restaurativa) (Chávez y García 2004: 92). Asimismo, se habrían incrementado los casos de adulterio - algunos autores dicen que por la mayor disponibilidad de tiempo de los hombres jóvenes (Chávez y García 2004: 203-204)—, resueltos igualmente por intermediación de los síndicos de las comunidades (Chávez y García 2004: 91). Y los conflictos de propiedad — sustracciones de bienes y cosechas, daños a la propiedad de un vecino, problemas de linderos, venta de tierras comunitarias, etc.generalmente solventados también por el síndico, que obliga al infractor a pagar el precio de lo sustraído y puede amenazarle con la expulsión del centro (Chávez y García 2004: 94). En Nangaritza, respecto a este tipo de casos, se suele hablar y negociar con el infractor alguna reparación, sin acudir a la autoridad estatal ni utilizar la amenaza del destierro. Nos llamó la atención, por ejemplo, la resolución tomada por distintos centros e impulsada por la FEPNASH en un caso de hurtos en las escuelas de las comunidades: se decidió coordinar un castigo, consistente en encerrar el mismo día y al mismo tiempo a los responsables de los robos (menores de edad) en una habitación de cada escuela (Presidente de la FEPNASH, entrevista personal, 6 sep. $2016)^{15}$.

Otros problemas son relativamente nuevos, fruto, en gran medida, del nuevo patrón de residencia. Por ejemplo, los relacionados con la falta de asunción de respon-

\footnotetext{
${ }^{14}$ Así lo aplican comunidades shuar de Morona Santiago, según Chávez y García (2004: 93). Los vecinos/as de las comunidades etnografiadas, en cambio, dicen no contemplar la medida de la expulsión.

15 En este y otros casos especialmente sensibles citados damos una identificación vaga de las comunidades para preservar el anonimato de los implicados.
} 
sabilidades comunitarias (no acudir a alguna tarea colectiva, a reuniones, etc.). Muchos centros incluyen en sus estatutos multas para castigarlo. Algunos de estos problemas se atribuyen, por parte de los propios shuar, a una pérdida de los valores tradicionales (Chávez y García 2004: 102; Nangaritza, entrevistas, sep. 2016); aunque lo significativo es que, al ser infracciones relativamente nuevas, los shuar no cuentan con precedentes para organizar su resolución (veremos, en efecto, que las dudas en torno a su sistema de justicia actual son numerosas, y que la utilización de la justicia estatal es habitual).

Conflictos nuevos son también los delitos de abuso sexual y abandono de responsabilidades conyugales — siendo nuevo no el tipo de fenómeno sino su consideración como infracción-. Los primeros se intentan resolver dentro de las comunidades y no suelen ser llevados a la justicia estatal. En una de las comunidades de Nangaritza una informante nos explicaba un caso de agresión sexual cometida en una fiesta por un joven ebrio. Los padres de víctima y agresor "negociaron": los de la agredida, proponiendo encerrar en un cuarto durante dos días al agresor; los del muchacho, negándose a ello por "no ser algo propio de los shuar" y prefiriendo, en cambio, que se macheteara al hijo, se le llevara a la cascada y se le hiciese ayunar. Finalmente se impuso esta solución y ambas partes llegaron a un acuerdo. En otra comunidad de Nangaritza una niña de 11 años sufrió abusos por parte de su tío, cuñado de su madre; esta se negó a denunciarlo ante la policía y silenció el asunto.

Para los casos de dejación de responsabilidades parentales, especialmente de reclamo de pensiones alimenticias o de asunción de la paternidad, sí es habitual el recurso a la justicia estatal, siendo el tipo de caso más frecuente en la Fiscalía de Asuntos Indígenas de Zumbi. Aunque sobre todo parecen llevarse allí como una forma de presionar a la expareja; la mayor parte de veces las mujeres acaban retirando la denuncia y no dan el paso de activar el rastreo policial del denunciado.

Este uso habitual del sistema de justicia estatal es, de hecho, otro cambio vital y complejo que ha sufrido la justicia shuar. Una forma, en realidad, de coordinación desde abajo: de uso selectivo de dos sistemas de justicia, para complementar o anular las decisiones respectivas (Santos 2012). Para algunos temas, de hecho, los shuar de Nangaritza declinan tener jurisdicción. Por ejemplo, para los homicidios y otros asuntos penales. La razón principal es cierto temor a la reacción del Estado: "Te pueden meter en la cárcel", comentaban en Yayu (entrevista grupal, 17 sep. 2016). No tiene que ver, por lo tanto, con el hecho de creer incompetente o inválida la forma tradicional de manejar estos casos. Chávez y García (2004: 93) consideran, de hecho, que entre los shuar "no se han podido desterrar de manera definitiva los conceptos tradicionales sobre la vida y la muerte, que consideran que una muerte solo se subsana con otra muerte". Veremos en el próximo apartado, no obstante, que las dudas y salvaguardas respecto a la acción del Estado condicionan el proyecto de reconocimiento y reactualización de la justicia shuar, y que la coordinación entre justicias (la indígena shuar y la estatal) es uno de los asuntos más espinosos.

Pero hay algunas variables que explicarían el que una comunidad concreta traslade más o menos casos a la justicia estatal: sobre todo, el grado de cohesión interna (Chávez y García 2004: 105); y la existencia, añadiríamos, de líderes fuertes que asuman el rol de jueces. En efecto, en las comunidades más cohesionadas — como alguna de las estudiadas en Nangaritza - se prefiere la resolución de los conflictos den- 
tro de la comunidad y tiene mala prensa, de hecho, trasladar los problemas a las instituciones del Estado. Además —y según nos comentaban los vecinos de Yayu-, hay razones concretas para preferir la justicia propia a la estatal: la mayor agilidad en la resolución de los procedimientos y el menor coste, por ejemplo. A partir de su experiencia personal, uno de los participantes en los talleres denunciaba que en la justicia estatal "todo se paga", y que allí "solo te cogen el dinero" (pueden leerse testimonios similares en Van Cott 2006: 212).

Por otro lado, comparar la situación y las percepciones entre comunidades resulta interesante, especialmente si es entre comunidades shuar y mestizas. Si en las primeras existen problemas y dudas a la hora de practicar $s u$ justicia, en las segundas se anhela un tipo de justicia propia, comunitaria, que solvente los problemas de convivencia cotidianos. Según se reveló en el taller realizado el 16 de septiembre de 2016, los vecinos de Nuevo Paraíso observan con envidia el hecho que los shuar vecinos hagan su propia justicia; "los nativos son más organizados", concluían.

\section{EXPECTATIVAS: EL INCIPIENTE PROCESO DE ESTATALIZACIÓN DE LA JUSTICIA SHUAR}

El deseo de los shuar de Nangaritza y de las organizaciones de la provincia es tener su propia justicia, autónoma y reconocida por el Estado: "la justicia shuar". Se entiende que algunos conflictos — como los reclamos de paternidad, de pensión alimenticia...- deberían ser tratados básicamente en las comunidades. Pues acudir a la justicia estatal crea un problema mayor. Que una mujer denuncie a su exmarido ante un juez estatal y haga que lo encarcelen, por ejemplo, solo consigue que el hombre "Cultive odio hacia ella" (Presidente FEPNASH, entrevista personal, 6 sep. 2016). En general, acudir a la policía o a los tribunales para solventar cualquier conflicto es juzgado negativamente, respondería al "facilitismo" de estos tiempos (Yayu, entrevista grupal, 17 sep. 2016).

Para revertir tal situación, los shuar de comunidades como Yayu creen que el primer paso es recuperar y valorizar las costumbres jurídicas ancestrales — se lamentan, de hecho, de que actualmente "se vaya poco a la cascada" o no se tomen las "plantas que dan poderes" (entrevista grupal, 17 septiembre 2016) ${ }^{16}$ —. A nivel institucional, la FEPNASH ha organizado una primera asamblea para discutir acerca de la estatalización de la justicia shuar (Presidente FEPNASH, entrevista personal, 6 sep. 2016).

Pero destacan en todo este proceso de recuperación y conformación de la justicia shuar dos fenómenos: las dudas comentadas respecto al encaje en el marco estatal y constitucional; y la tutela por parte de los kichwas saraguro.

Respecto al primer punto, la mayor parte de los shuar con los que hemos hablado cree que su justicia servirá para ocuparse "de las pequeñas cosas", de los problemas cotidianos de las comunidades. De otros asuntos, "la Constitución no permite"

\footnotetext{
${ }^{16}$ No parece cumplirse, en el caso de comunidades como Yayu, la tesis de Grijalva respecto al desinterés en la justicia indígena por parte de los jóvenes (Grijalva 2012b: 568). En Yayu son hombres jóvenes o de mediana edad quienes participan más activamente en talleres como el que organizamos, dedicados a la recuperación de la justicia ancestral shuar.
} 
(Yayu, entrevista grupal, 17 septiembre 2016; Presidente FEPNASH, entrevista personal, 6 sep. 2016). Se da, así, una especie de autocensura y cierto temor a infringir lo dispuesto por la Constitución - y desatar con ello la punición del Estado- (ver Van Cott 2006: 211). En el caso de las retaliaciones tradicionales, de la sanción de muerte a un homicida, todos parecen estar de acuerdo en que hoy resulta imposible de aplicar (la propia Federación Interprovincial —FICSH—, como veíamos, renunció a ella formalmente y la substituyó por la expulsión de las comunidades) (Chávez y García 2004: 202).

Como se indicaba en la introducción, el gran tema pendiente es el de la coordinación entre las justicias indígenas y la estatal, dado que en esta última los delitos, sanciones y procedimientos propios de las culturas ancestrales no parecen comprenderse del todo (Stavenhagen 2006). En el caso de los shuar, su cultura jurídica contempla — como se ha descrito- numerosos castigos físicos. Aunque estos tienen como objetivo purificar y reintegrar en la comunidad al malhechor, la justicia estatal los juzga como atentados intolerables a la dignidad de la persona (Grijalva y Exeni 2012). En Yayu, conscientes de ello, nos comentaban que hoy es imposible machetear a un adúltero como se hacía antaño, pues los acusarían "de no respetar sus derechos humanos" (entrevista grupal, 17 sep. 2016).

Otro problema distinto de coordinación es el que se da en las comunidades interétnicas, entre las distintas justicias indígenas. Los shuar de Nangaritza se interrogan sobre situaciones que ya se están produciendo en sus comunidades: «ahí, digamos, un saraguro con un shuar. Cómo se va a coordinar, ¿aplicar una sola sanción para los dos o de manera separada cada uno su sanción?..., nos falta muchísimo" (Daniel Chuinda, expresidente FEPNASH, entrevista personal, 26 may. 2016). Y censuran, en general, que se asuman maneras de hacer que no corresponden a las (supuestas) costumbres shuar, añadiendo para fundamentar la crítica el conveniente respeto al marco estatal:

Están haciendo de manera que no está permitida por la Constitución, por los Derechos Humanos... Entonces claro, ellos [los shuar] a lo mejor harán lo que no hacían, hacen lo del saraguro, por ejemplo; un compañero de la comunidad dice "te voy a aplicar esto", y dice "lo chinen", por ejemplo. Ya empezaron a chinar, pero eso es como aplican los saraguro (...) Les hice una observación: "compañeros, eso no es nuestra forma de sancionar; qué hacen, estamos copiando lo ajeno, por qué no empezamos con lo nuestro" (Daniel Chuinda, entrevista personal, 26 may. 2016).

Desde la perspectiva saraguro, en cambio, se censura que algunos shuar prefieran recurrir a la justicia estatal antes que a la indígena (aunque no sea la propia):

Algunos hermanos shuar, algunos no han querido someterse a la justicia indígena saraguro, "yo tengo mi abogado, me han planteado una serie de argumentos...", pero a la final los jueces y fiscales han dicho "está en manos de los jueces saraguro" (Presidente ZAMASKIJAT, entrevista personal, 27 ago. 2016).

Precisamente, volviendo a la cuestión del reconocimiento estatal de la justicia shuar, el segundo punto que indicábamos era la tutela por parte de los saraguro. Es un hecho que el pueblo saraguro ha avanzado considerablemente en la oficialización de su propia justicia. Por ejemplo, ha sistematizado ya en una publicación las distintas instancias y foros de coordinación que se desea establecer (GAD Provincial Zamora 
Chinchipe 2016). Son saraguro quienes encabezan organismos como la Unidad de Interculturalidad de la Prefectura de Zamora, encargadas de comandar el reconocimiento de las justicias indígenas. Y las organizaciones shuar buscan la guía de los saraguro para llevar a cabo su propio proceso (líderes saraguro dirigieron, de hecho, la primera asamblea sobre justicia shuar).

Pero cabe señalar que la justicia saraguro oficializada parece inspirarse notablemente en la estatal, mimetiza su lenguaje y estructura (Grijalva 2012b). Por ejemplo, establece distintas instancias de apelación, siendo la primera la Asamblea Comunitaria, la segunda la Asamblea de Autoridades de la parroquia, y la tercera y cuarta la Asamblea de Autoridades del cantón y de la provincia, respectivamente (GAD Provincial Zamora Chinchipe 2016) ${ }^{17}$. Y los dirigentes de organizaciones saraguro como ZAMASKIJAT (la Federación Provincial de la Nacionalidad Kichwa Saraguro en Zamora) manifiestan que la idea es replicar el modelo para el caso shuar (entrevista, 27 sep. 2016). Algo que no debería ser difícil, pues, como veíamos, los shuar también contemplan hoy distintos niveles de justicia:

Nosotros hemos dicho primero la comunidad, y si no pueden resolver con las autoridades de la comunidad, tienen que ir a la autoridad de la Asociación, porque también hay asociaciones; y luego va así, en orden jerárquico, a la Federación (Expresidente FEPNASH, entrevista personal, 26 may. 2016).

No obstante, sí se prevén algunas complicaciones. Por ejemplo, en cuanto al alcance y competencias de la justicia indígena. Al contrario que los shuar, que muestran cierta contención y dudas, los saraguro explicitan una propuesta de alcance maximalista (su justicia sería competente en todo tipo de casos); lo que ya ha comportado problemas con las autoridades estatales. Explicaba el presidente de ZAMASKIJAT:

No hay límite de los casos que podamos agarrar. Que nos digan que casito más pequeño nomás ustedes pueden resolver. Nosotros hemos agarrado todos los casos que han sido puestos en conocimiento de la coordinación provincial (...). Nosotros nos hemos acogido a sentenciar directamente nosotros, y nosotros pasamos ya la sentencia a la Fiscalía o a las Cortes, para que se archive el proceso, porque ya es caso juzgado; pero cuando cumpla el trabajo comunitario de los cinco o seis años [el sentenciado]... (...). De repente algunos compañeros dicen, "como ya está archivado", ellos salen nomás de la comunidad, se pasean por la ciudad... Tenemos un compañero que está en San Vicente, por ejemplo, de repente lo ven en el terminal, el presidente lo ve y le dice "cuidado que vas a ser detenido", y se va y se pierde (Presidente ZAMASKIJAT, entrevista, 27 sep. 2016).

Por otro lado, los propios Saraguro mantienen dudas respecto a la adaptabilidad de la justicia shuar al marco estatal, juzgándola más arcaica:

Porque ellos son, para solucionar, un poco más duros, drásticos, inclusive; por el tema de tsansa [toma y reducción de cabezas de enemigos] y las guerras internas que había. Pero como la Constitución no permite la violación de derechos humanos, entonces también ellos tienen que entender esa parte, porque ya es inconstitucional (Presidente ZAMASKIJAT, entrevista personal, 27 ag. 2016).

\footnotetext{
${ }^{17}$ No obstante, otros elementos parecen alejarse de la justicia estatal. Por ejemplo, la distinción entre autoridades revocables y permanentes, siendo estas últimas — más importantes - los padrinos y líderes reconocidos de cada comunidad (imbricación con la vida social muy propia de los sistemas vindicatorios).
} 
En lo que parece una recreación de algunos tópicos sobre el pueblo shuar, y de ciertas incomprensiones en torno a su justicia vindicatoria. Y ello a pesar de que los saraguro tienen una justicia de base igualmente vindicatoria, que contempla ofensas, negociación entre las familias y restauración de los vínculos sociales —aunque con otras normas y procederes-.

\section{APUNTES FINALES}

El proyecto de estatalización de la justicia shuar se antoja complicado, tratándose de un sistema típicamente vindicatorio (incomprendido incluso entre los especialistas en cultura shuar y justicias indígenas) y observando las dificultades que hay para que se acepten elementos como el castigo físico o las retaliaciones. Una hipótesis plausible es que la justicia shuar oficializada acabe perdiendo gran parte de su carácter original.

Esto, por lo demás, ha ocurrido con otros sistemas de justicia indígenas reconocidos por el Estado. Caracterizados por la flexibilidad de sus procedimientos, por la adaptación al caso o la imbricación entre el ámbito de lo jurídico y de lo social, en general, el reconocimiento ha comportado frecuentemente la sustracción de parte de esos rasgos. Al tiempo que la codificación - o creación de una versión oficial-, ha supuesto en ocasiones una pérdida de legitimidad de esas justicias entre sus antiguos practicantes (Grijalva 2012b: 566-567; Stavenhagen 2006: 221). Aunque mimetizar el lenguaje y estructura del derecho estatal — como parecen estar haciendo los saraguro de Zamora Chinchipe- parece una estrategia para conseguir la aprobación del Estado, esto lleva frecuentemente a la desfiguración comentada. Ha pasado en casos como el tsachila, donde el proceso de estatalización significó, básicamente, la asunción de una racionalidad técnico-burocrática extraña para los tsachila, que transformó su derecho propio en algo de difícil comprensión y a la postre ineficaz (Fiallo 2014: 107-111).

Relacionado con esto, uno de los grandes retos para las justicias indígenas en general es la coordinación con la justicia estatal. Un proyecto de Estado plurinacional e interétnico como el ecuatoriano aspira a un pluralismo jurídico real, en el que los distintos sistemas de justicia se reconozcan mutuamente y convivan en igualdad (Santos 2012: 36-37). Algunos pasos en este camino sí parecen haberse dado, con cierta disposición de algunos operadores de la justicia estatal a delegar en las autoridades indígenas los problemas que afectan a las comunidades (así lo manifiestan los representantes saraguro de Zamora). No obstante, y a la espera de una ley que regule formalmente estos trasvases, el vacío legal hace prever serios conflictos.

Por el momento, los shuar de Nangaritza parecen mantener una relación de complementariedad con el sistema estatal. Hacen uso de él estratégicamente, para validar algunas actuaciones en el ámbito comunitario y familiar, por ejemplo; rehuyen tratar otros asuntos en la justicia estatal, en cambio, y sí asumen que para ciertos conflictos no tienen competencias. Pero la definición de las jurisdicciones es también para ellos una cuestión controvertida, a resolver. Algunos líderes parecen reservarle a la justicia shuar los asuntos menores, considerándola apta solo para las pequeñas ofensas y rencillas cotidianas. Mientras que para otros debería poder ocuparse de 
cualquier caso, incluidos (utilizando las categorías estatales) los de ámbito penal - sobre los homicidios, no obstante, hay unanimidad en considerar que no se podrán tratar-.

Mientras tanto, líderes y vecinos shuar del cantón Nangaritza siguen movilizándose e investigando sobre su cultura, intentando encontrar las bases con que negociar el establecimiento de su propia justicia. En un proceso que se antoja largo y tortuoso.

\section{BIBLIOGRAFÍA CITADA}

Aparicio, Marco. 2015. "Derechos y pueblos indígenas: avances objetivos, debilidades subjetivas". Revista de Antropología Social 24: 127-147.

Ávila, Laura F. 2012. "Disputas de poder y justicia: San Lucas (Saraguro)", en Boaventura S. Santos y Alejandro Grijalva (eds.), Justicia indigena, plurinacionalidad e interculturalidad en Ecuador. 373-430. Quito: Abya Yala/Fundación Rosa Luxemburg.

Brandt, Hans-Jürgen y Rocío Franco (eds.) 2007. Normas, valores y procedimientos en la justicia comunitaria: estudio cualitativo en comunidades indígenas y campesinas de Ecuador y Perú, vol. II de Justicia comunitaria en los Andes: Perú y Ecuador. Lima: Instituto de Defensa Legal.

Chávez, Gina y Fernando García. 2004. El derecho a ser: diversidad, identidad y cambio. Etnografía jurídica indígenas y afroecuatoriana. Quito: FLACSO.

Clastres, Pierre. 1978. La sociedad contra el Estado. Barcelona: Monte Ávila.

Fiallo, Liliam. 2014. Pluralismo jurídico en Ecuador: El caso de la nacionalidad Tsachila. Quito: Universidad de las Américas.

GAD Cantonal Nangaritza. 2015. Plan de Desarrollo y Ordenamiento Territorial. Guayzimi: Gobierno Autónomo Descentralizado del cantón Nangaritza.

GAD Parroquial Nuevo Paraíso. 2015. Plan de Desarrollo y Ordenamiento Territorial Parroquial de Nuevo Paraíso, 2015-2030. Nuevo Paraíso: Gobierno Autónomo Descentralizado de la parroquia Nuevo Paraíso.

GAD Provincial Zamora Chinchipe. 2015. Plan de Desarrollo y Ordenamiento Territorial 2015-2019. Zamora: Gobierno Autónomo Descentralizado Provincial de Zamora Chinchipe.

GAD Provincial Zamora Chinchipe. 2016. Instituciones y Autoridades de Justicia Indígena de la Nacionalidad Kichwa. Zamora: Gobierno Autónomo Descentralizado Provincial de Zamora Chinchipe.

García, Fernando. 2002. Formas indígenas de administrar justicia. Estudios de caso de la nacionalidad quichua ecuatoriana. Quito: Flacso.

Grijalva, Agustín. 2012a. "Del presente se inventa el futuro: justicias indígenas y Estado en Ecuador", en Boaventura S. Santos y Alejandro Grijalva (eds.), Justicia indigena, plurinacionalidad e interculturalidad en Ecuador. 51-77. Quito: Abya Yala/Fundación Rosa Luxemburg.

Grijalva, Agustín. 2012b. "Conclusiones de todos los estudios: experiencias diversas y convergentes de la justicia indígena en Ecuador", en Boaventura S. Santos y Alejandro Grijalva (eds.), Justicia indigena, plurinacionalidad e interculturalidad en Ecuador. 551-577. Quito: Abya Yala/Fundación Rosa Luxemburg.

Grijalva, Agustín y José Luis Exeni. 2012. "Coordinación entre justicias, ese desafío”, en Boaventura S. Santos y Alejandro Grijalva (eds.), Justicia indígena, plurinacionalidad e interculturalidad en Ecuador: 581-613. Quito: Abya Yala/Fundación Rosa Luxemburg.

Harner, Michael. 1972. The Jivaro. People of the Sacred Waterfalls. Nueva York: Anchor Press.

Karsten, Raphael. 1988 [1935]. La vida y la cultura de los shuar. Guayaquil: Abya Yala.

Llasag, Raúl. 2012. "Justicia indígena, ¿̇delito o construcción de la plurinacionalidad?. La Cocha", en Boaventura S. Santos y Alejandro Grijalva (eds.), Justicia indigena, plurinacionalidad e interculturalidad en Ecuador: 321-372. Quito: Abya Yala/Fundación Rosa Luxemburg.

Martínez Novo, Carmen. 2014. "Managing Diversity in Postneoliberal Ecuador”. The Journal of Latin American and Caribbean Anthropology 19(1): 103-125. 
Moore, Sally F. 1972. "Law and Social Change. The Semi-autonomous social field as an appropriate subject of study". Law \& Society Review 7(1): 719-746.

Observatorio de Conflictos Socioambientales. 2013. Territorio shuar: Entre sobrevivencia y protección ambiental en el Alto Nangaritza. Loja: Ediloja.

Roseberry, William. 2014. Antropologías e Historias. Ensayos sobre cultura, bistoria y economía politica. Zamora: El Colegio de Michoacán.

Rubenstein, Steve. 2005. "La conversión de los shuar". Íconos. Revista de Ciencias Sociales 22: 27-48. doi: <https://doi.org/10.17141/iconos.22.2005.99>.

Santos, Boaventura S. 2012. "Cuando los excluidos tienen Derecho: justicia indígena, plurinacionalidad e interculturalidad", en Boaventura S. Santos y Alejandro Grijalva (eds.), Justicia indigena, plurinacionalidad e interculturalidad en Ecuador: 13-50. Quito: Abya Yala/Fundación Rosa Luxemburg.

Sierra, María Teresa y Victoria Chenaut. 2006. "Los debates recientes y actuales en la Antropología Jurídica: las corrientes anglosajonas", en Laura R. Valladares (ed.), V Congreso de la Red Latinoamericana de Antropología Jurídica: 27-58. Oaxtepec: Universidad Autónoma de México. Disponible en: <http://www.dfpd.edu.uy/departamentos/sociologia/adjuntos/jornada_6_abril/ Antropolog\%C2\%A1a_jur\%C2\%A1dica.pdf>. Fecha de acceso: 23 ene. 2016.

Stavenhagen, Rodolfo. 2006. "Derecho consuetudinario indígena en América Latina", en Laura R. Valladares (ed.), V Congreso de la Red Latinoamericana de Antropología Jurídica: 15-26. Oaxtepec: Universidad Autónoma de México. Disponible en: <http://www.dfpd.edu.uy/departamentos/ sociologia/adjuntos/jornada_6_abril/Antropolog\%C2\%A1a_jur\%C2\%A1dica.pdf>. Fecha de acceso: 23 ene. 2016.

Terradas, Ignasi. 2008. Justicia vindicatoria. Madrid: CSIC.

Terradas, Ignasi. 2012. "Què és la justícia vindicatòria? (Definició i característiques fonamentals)". Recerques 64: 13-30.

Trujillo, Julio César. 2012. "Plurinacionalidad y Constitución", en Boaventura S. Santos y Alejandro Grijalva (eds.), Justicia indigena, plurinacionalidad e interculturalidad en Ecuador: 305-314. Quito: Abya Yala/Fundación Rosa Luxemburg.

Van Cott, Donna Lee. 2006. "Pluralismo legal y administración de justicia comunitaria informal en América Latina", en Laura R. Valladares (ed.), V Congreso de la Red Latinoamericana de Antropología Jurídica: 209-238. Oaxtepec: Universidad Autónoma de México. Disponible en: <http://www.dfpd.edu.uy/departamentos/sociologia/adjuntos/jornada_6_abril/Antropolog\%C2\%A1a_jur\%C2\%A1dica.pdf >. Fecha de acceso: 23 ene. 2016.

Vintimilla, Jaime, Milena Almeida y Remigia Saldaña (eds.). 2007. Justicia comunitaria en los Andes: Perú y Ecuador (vol.IV). Derecho indígena, conflicto y justicia comunitaria en comunidades Kichwa del Ecuador. Lima: Instituto de Defensa Legal.

Wolkmer, Antonio C. 1994. Pluralismo jurídico. Fundamentos de uma nova cultura no direito. San Pablo: Alfa Omega.

Fecha de recepción: 23 de enero de 2017

Fecha de aceptación: 24 de julio de 2017 